\title{
WHITHER FLAT PANEL DISPLAYS?
}

\author{
Kala Krishna \\ Marie Thursby
}

Working Paper 5415

\author{
NATIONAL BUREAU OF ECONOMIC RESEARCH \\ 1050 Massachusetts Avenue \\ Cambridge, MA 02138 \\ January 1996
}

This paper is part of NBER's research program in International Trade and Investment. Any opinions expressed are those of the authors and not those of the National Bureau of Economic Research.

(C) 1996 by Kala Krishna and Marie Thursby. All rights reserved. Short sections of text, not to exceed two paragraphs, may be quoted without explicit permission provided that full credit, including $\odot$ notice, is given to the source. 


\title{
WHITHER FLAT PANEL DISPLAYS?
}

\begin{abstract}
This paper examines possible consequences of subsidies to R\&D and to volume production proposed under the Clinton administration's flat panel display initiative. We do this in the context of a model in which firms behave competitively in the short run, while realizing that their choices of capacity and yield-improving R\&D in the medium and long run will affect market price. Policy simulations show that steady state yields and profits are lower, while prices are higher with subsidies for capacity acquisition than with R\&D subsidies. This occurs because a firm's incentives to do R\&D are diminished by a subsidy on capacity costs.
\end{abstract}

Kala Krishna

Department of Economics

The Pennsylvania State University

Kern Graduate Building

University Park, PA 16802-3306

and NBER
Marie Thursby

Department of Economics

Purdue University

1310 Krannert Building

West Lafayette, IN 47907-1310

and NBER 


\section{Introduction}

In April 1994, the Clinton administration announced a $\$ 597$ million policy initiative to encourage U.S. companies to invest in research and development $(R \& D)$, as well as large scale production of flat panel displays $(F P D)$. Semiconductors have become a standard part of many manufactured products and flat panel displays are seen as having a similar potential. In the near future, however, computers are likely to account for more than half of $F P D$ demand. Recommended support for the National Flat Panel Display Initiative included $\$ 318$ million for core research and development $(R \& D)$, $\$ 50$ million for a manufacturing test bed, $\$ 199$ million for $R \& D$ linked to volume production, and $\$ 20$ million for procurement incentives. Among the initiative's goals is the creation of a U.S.-based industry capable of achieving a 15 percent share of the world FPD market by the year 2000 (Flamm 1994).

The initiative has been highly controversial. ${ }^{1}$ While the Department of Defense $(D o D)$ claims domestic production of FPDs is critical for national security, critics label the initiative as misguided industrial policy. Citing surveillance experiences from Desert Storm and the growing importance of digital technologies, $D o D$ officials argue that effective use of advanced information technologies will determine the winners of future military conflicts. Visual displays are seen as a critical factor in this regard. Moreover, relative to cathode ray tube displays, FPDs are reliable, lightweight, and energy efficient. Thus, $D o D$ justifies the need for policy by citing limited U.S. capacity to produce $F P D s$ and the apparent refusal of Japanese companies to tailor FPDs to $D o D$ specifications (DoD 1994).

Critics of the initiative argue that U.S. military demand for FPDs could be met easily by $\overline{1 \text { See Barfield } 1994 \text { and 1995, Flamm } 1994}$ and 1995, Miller 1995, and Mowery 1995. 
several small to medium size plants, an increase in capacity much less than that required to capture 15 percent of the world market (Barfield 1994, 1995; Mently 1994b). DoD estimates of future military demand for FPDs are 15,000 annually from $1995-1999$ and 25,000 annually from $2000-2009$ ( $D o D$ 1994, page $I I I-14$ ). Thus, even $D o D$ estimates place defense needs for FPDs far below the initiative's goal of 15 percent of the world market. It is not surprising, then, that the initiative is considered by some to be thinly disguised industrial policy. In addition, it is also argued that the size of the initiative is too small for it to make much of a difference.

Viewed as such, a question of interest becomes whether the initiative will lead to an increase in output, and whether these firms will earn economic rents. ${ }^{2}$ Although this question has not been directly addressed, Mently's market projections suggest the initiative's output effects, per se, will be small relative to its goal. He claims a 15 percent market share for U.S. companies would require an investment of $\$ 3$ billion, rather than the planned $\$ 600$ million. Mently's projected rates of return for active matrix liquid crystal displays also suggest relatively low profit margins beginning in 1995 (Mently 1995). ${ }^{3}$

Another point of controversy is whether the initiative is consistent with post-Uruguay Round subsidy codes. Since $R \& D$ support under the initiative is limited to 50 percent of project cost, $D o D$ officials argue it is well within the 75 percent cap for subsidies for specific industrial research. Critics, such as Barfield, argue that the incentives for volume production are beyond precompetitive support and that the procurement incentives directly violate the new GATT codes. There are, however, exceptions to these rules for defense.

In this paper, we consider a related set of issues, namely the impact of subsidies for core 2 Of course, an important issue here is whether profit increases and/or spillover effects are sufficient to increase national welfare.

${ }^{3}$ Lower profit margins are a result, in part, of increased world capacity with the recent entry by Korean firms (Pollack 1995). 
$R \& D$ and of subsidies targeted to high volume production. We do this in the context of a model which we feel captures the importance of investment in capacity, as well as yieldimproving $R \& D$, in the flat panel industry. In this model, firms with higher yields, ceteris paribus, invest in more capacity than those with lower yields. This leads to higher capacity and output for high yield firms. The model has the property that all firms who remain in the industry ultimately have the same yield, i.e. in the steady state. Thus firms with an initial advantage in terms of yields tend to remain ahead for a while, after which laggards catch up. However, note this gives firms with higher initial yields a stream of quasi-rents, which the laggards do not enjoy. Such characteristics are thought to apply to industries such as FPDs and semiconductors.

Relevant industry characteristics are discussed in Section 2, and a simple, stylized model is presented in Section 3. In Section 4, we examine the fit between the cost structure postulated in the model and data on manufacturing costs for color thin film transistor liquid crystal displays $(T F T-L C D \mathrm{~s})$. Policy simulations are presented in Section 5 . We first look at a permanent $25 \%$ subsidy on capacity acquisition versus a $25 \%$ subsidy on yield improving $R \& D$. Our results suggest that capacity subsidies have the unfortunate effect of decreasing incentives for $R \& D .{ }^{4}$ This, in turn, leads the targeted firm to have lower steady state yields with capacity subsidies than with either $R \& D$ subsidies or in the absence of policy.

We then compare the effects of a one shot fixed expenditure by the government on capacity and $R \& D$ subsidies. This gives us an idea of the difference in leverage provided by

4 Care should be taken in relating the complex and difficult to implement proposals by the defense department to the simpler policies discussed here. The $D o D(1994$, page $I-9)$ states that "selected companies commited to new investments in volume production facilities for current generation products would be eligible to receive $R \& D$ support for next generation products and manufacturing processes, commensurate with the level of commitment demonstrated to volume production." This makes the subsidy conditional on capacity acquisition, so that it can be interpreted as, in part, a capacity subsidy. 
the two instruments since the subsidies are specified as a given dollar amount. We find that this type of subsidy has a positive impact effect on the net $R \& D$ and capacity expenditure by firms when the subsidy is for $R \& D$, but not when it is a capacity subsidy! In our model, $R \& D$ subsidies tend to provide more leverage than capacity subsidies. Directions for future work are discussed in Section 6.

\section{The Industry}

The flat panel industry provides an interesting case study, quite apart from the $D o D$ initiative. It is a highly concentrated industry, in which survival depends on continual investment in both product and process $R \& D$. Much like other high-technology electronics products, there are a variety of rival technologies undergoing development. Successful development of one technology does not guarantee profits for long because new technologies and new generations of old technologies are constantly being developed.

There are a multitude of interesting characteristics of the industry, and it is impossible to develop one model which captures all of them. Our model captures the idea that continual innovation is needed to reap profits. If a firm does not improve its yield, it falls behind. As the yields of its competitors rise, market price declines, eventually wiping out the lagging firm's profits, at which point it exits. In focusing on the role of yield-improving innovation, we take several shortcuts. We assume that firms are concerned only with the current cycle of production, capacity acquisition and $R \& D$. They do not look forward to anticipate the impact of their behavior today on future cycles. We choose to do this because uncertainty about the future is high in this industry, making standard dynamic approaches ill-suited for modelling it. In the interest of simplicity, we also neglect other features such as adjustment costs even though they are important in the industry. Capacity costs are likely to have a 
high sunk component leading to irreversibilities and adjustment costs. Adjustment costs of this kind create "hysteresis," so that history matters. This creates situations where a temporary policy or shock can have permanent effects by causing what looks like a regime change..$^{5}$

We abstract from several other factors, which while important in the industry, have been studied elsewhere. Thus, including these factors would add little that is not already well understood. For example, some of the yield improvement observed in the industry is likely to result from learning by doing. Models of learning by doing are well studied in the literature. In a trade context, Baldwin and Krugman (1988) model the semiconductor industry in this manner. One result from such models is that firms produce more than they would if they equated marginal revenue and cost since they take into account the reduction in future costs implied by an additional unit of current output. In such contexts, capacity subsidies are in effect also $R \& D$ subsidies, making these models ill-suited for answering the kind of questions we ask. That is, in learning by doing models, stark differences between $R \& D$ and capacity subsidies cannot be detected. We also assume there are no spillovers in $R \& D$. Such spillovers occur when a yield improvement by one firm accrues, at least partially, to other firms. Again, it is well understood, ${ }^{6}$ that spillovers of this kind reduce the ability to internalize the effects of $R \& D$ and hence tend to reduce the incentive to do $R \& D$.

\subsection{FPD Technologies}

As shown in Table 1, liquid crystal displays $(L C D)$ are the major type of display in today's market, comprising 87 percent of the commercial market. In these displays, light is emitted when voltage is applied to liquid crystals enclosed between the two sheets of glass (substrates)

6 For an analysis of $R \& D$ spillovers in an international trade context, see Jensen and Thursby (1987). 
that make up the display. ${ }^{7}$ Active matrix $L C D s(A M L C D s)$, which control the polarization of these crystals by use of silicon transistors, are considered the dominant technology for high information displays. The primary use of $A M L C D s$ is color screens for laptop computers. Passive matrix $L C D s$ (PMLCD) control light emission by use of metal horizontal and vertical electrodes on the two sheets of glass. PMLCDs are more commonly used than $A M L C D s$, but they have slower response times and tend to be less bright ( $D o D$ 1994). Hence, as noted in the table, $D o D$ predicts an increase in the importance of $A M L C D s$, relative to $P M L C D s$.

One problem with $L C D s$ is that even with redundant transistors at each pixel, some pixels fail to operate with resulting quality problems in the display. $L C D s$ are produced under the same clean room conditions as semiconductors, and displays which contain defective pixels risk being discarded. This means quality control problems increase dramatically as the screen size increases, so that the potential of $L C D s$ for large screen applications is limited. Moreover, $L C D$ producers engage in $R \& D$ to develop new generations of equipment which are capable of producing higher yields, i.e., portion of production which is of acceptable in terms of defective pixels.

Three alternatives to $L C D s$ are plasma displays $(P D P)$, electroluminescent displays $(E L D s)$, and field emission displays $(F E D)$. These displays differ from $L C D s$ in that they produce their own light. $P D P s$ generate light by applying voltage to an inert gas enclosed between the two sheets of glass, while ELDs and FEDs stimulate phosphors to emit light. Notice these displays comprised only 5 percent of the market in 1993 , and $D o D^{\prime} s 1994$ report predicted emissive displays will remain a small part of the commercial market in 2000 . However, in August 1995, a number of companies announced progress toward development and production of PDPs and FEDs (Business Week 1995, Patton and Rawsthorn 1995). 7 See DoD (1994), Chapter II for a detailed description of each of the FPD technologies. 
Fujitsu, Matsushita, Sony, and $N E C$ unveiled working prototypes of large screen PDPs (up to 42 inches), and Fujitsu announced plans to produce 10,000 units a month by October 1996. $P D P s$ are considered to have the greatest potential for low cost, large $T V$ screens. $^{8}$

\subsection{Market Shares in FPDs}

As with $V C R s$, the technologies leading to FPDs were developed in the labs of U.S. electronics companies, but the industry is now dominated by Japanese companies. ${ }^{9}$ For example, early technologies for $F P D s$ were developed in research labs at $R C A$, Westinghouse, and $I B M$ in the $1960 s$ and $1970 s$. Engineers in $R C A^{\prime} s$ Sarnoff Lab developed $L C D$ technology with the goal of developing a $T V$ screen "flat enough to hang on a wall" (DoD 1994, Ch.VI). Dr. Peter Brody, of Westinghouse, developed the cadmium selenide AMLCD technology underlying the majority of today's $A M L C D s$. Although these technologies were at the prototype stage, neither RCA nor Westinghouse pursued commercial development of $F P D$ s. $R C A$ switched from $L C D$ to cathode ray research in the 1970 s, while Westinghouse dropped its project in 1979. In the 1970s, IBM pursued plasma technologies for computers and business applications, but in 1984 it switched $\mathrm{R} \& \mathrm{D}$ efforts to $L C D$ technologies. In the late 1980s, IBM and Toshiba formed Display Technologies, Inc. (DTI), a joint venture for development and high volume production of $L C D \mathrm{~s}$ in Japan. None of these companies invested in manufacturing capacity in the United States. ${ }^{10}$

\footnotetext{
8 Notice in Table 1, the primary application of FPDs has been computers, with the consumer market representing only 12 percent of demand. While DoD predicts a small consumer market in 2000 , the evolution of cheaper large scale FPDs is likely to dramatically increase the consumer market.

9 The patent underlying virtually all VCR technologies was obtained by Ampex Corporation, a U.S. company, in the late 1950s. Although Ampex successfully commercialized a video tape recorder for broadcast use, the first companies to commercialize a consumer product were Sony, JVC, and Matsushita. This occurred despite early efforts by RCA to develop a consumer videorecorder and a joint-venture by Ampex and Toshiba for the same purpose. See Tyson (1992) for an analysis which focuses on the role of government policy in VCR development. In contrast, Rosenbloom and Cusumano (1987) take the view that the evolution of the industry is due primarily to different management styles of the companies involved.

${ }^{10 S e e} \mathrm{Ch}$. VI of the $1994 \mathrm{DoD}$ report for an account of other efforts by U.S., European, and Japanese companies.
} 
In contrast, companies such as Sharp and NEC actively pursued development and production of $F P D$ s. Both companies stood to benefit from their experience with the types of clean room conditions and continual process improvements important in semiconductors. Sharp successfully applied $F P D$ technology to hand-held calculators in the 1970 s, and in the 1980s it became a leader in color thin-film transistor $L C D$ s $(T F T-L C D)$. In 1993, Sharp was the world's largest producer of $F P D$ s, with its sales comprising 44 percent of the world market. NEC and DTI were the next largest producers, with sales comprising 35 percent of the market (DoD1994,Ch.IV).

In total, approximately 50 companies manufacture $F P D$ s. As noted in Table 2, Japanese companies accounted for over 90 percent of the $L C D$ market in 1993. Seven Japanese companies accounted for 98 percent of the $A M L C D$ market. In 1993, the only volume producer of $A M L C D$ s in the United States was Optical Imaging Systems, which primarily sold displays for avionics applications. If one views IBM's joint venture, DTI, as a Japanese company, the only markets in which U.S. companies are large players are the PDP and $E L D$ markets. The fact that these markets comprise only 5 percent of the total value of $L C D$ s (Table 1) is one aspect of $D o D$ 's justification for the flat panel policy initiative.

Several U.S. companies are conducting R\&D on $F P D$ s, in part, as a response to the $D o D$ initiative. Optical Imaging Systems was awarded an Advanced Research Projects Agency $(A R P A)$ contract to construct a new $A M L C D$ plant, and it is involved in a joint venture with Apple to develop displays for computer applications. Additionally, a consortium formed by AT\&T, Standish Industries, and Xerox received a $D o D$ award to develop a manufacturing test bed. Various other R\&D projects are ongoing at Motorola, Raytheon, Sarnoff Labs, and Texas Instruments. 


\section{A Simple Model}

In this section, we develop a simple model which we feel captures several important features of the flat panel display industry. First, we feel the timing structure needs to recognize that output is easier to change than capacity, which in turn is easier to change than yield. We therefore assume that in what we call the short run, both capacity and yield are fixed. In what we call the medium run, capacity is variable, and in the long run, yield can be chosen. Of course, in the steady state of such a model, long run and short run yields must be equal.

Second, we feel that in the short run, with capacity and yield as given, firms behave competitively and take price as given. Thus, their only decision is whether to produce to capacity or not. ${ }^{11}$ If profits are nonnegative, firms produce; and, if profits are negative, firms do not produce. However, in the medium run, they choose capacity. In making this decision, they take into account the fact that capacity affects output and hence profit. This results in a capacity choice equilibrium resembling a Cournot Nash equilibrium. In the long run, firms choose $R \& D$ expenditure which affects their yields, and hence their capacity choice, as well as output. Notice that we abstract from $\mathrm{R} \& \mathrm{D}$ to develop new types of $F P D \mathrm{~s}$, so that the only $R \& D$ is for process improvement.

Third, firms with a high yield find it easier to attain a given yield higher than their existing one than do firms with lower yields. However, any improvement in yields is costly. It is also assumed that there are positive costs of any improvement in yields, no matter how infinitesimal. The latter assumption permits convergence of the yields to a finite level in some steady states.

We wish to use the model to answer questions about the evolution of industry capacity.

11The clever reader will immediately think of a counterexample where the last firm's capacity, if used entirely, results in negative profits, but, if not used at all, results in a price higher than short run marginal cost. It is argued below that this case will never arise. The argument consists of showing that capacity choices of this kind are never made. 
If, for example, a given number of firms start off with different yields, what should we expect for their capacity and yield paths over time? Do firms that have better yields invest more or less in R\&D? Do firms with better yields stay ahead in terms of yields? If there is entry into the industry, will profits be eroded to zero? Does the final profile of an industry depend on the initial profile? How?

By examining these questions, we hope to shed light on the effect of policies designed to encourage investment in $R \& D$ and in production facilities. We find that firms with higher yields, ceteris paribus, invest in more capacity than those with lower yields. Since firms produce up to capacity, this implies more output by these firms. Higher outputs, in turn, increase the benefits from investments in $R \& D$ to raise yields. As our simulation results show, this conflagration of forces plays a role in the effects of capacity and R\&D subsidies. While capacity subsidies initially lead to increased capacity, and hence investment in yields, at the margin they reduce the benefit from yield-increasing $R \& D$. As shown in Section 4, this can lead to steady state yields which are lower than what would occur without the capacity subsidies. In contrast, subsidies to R\&D promote higher steady state yields.

\subsection{The Short Run}

Assume that there are $N$ firms in the industry. Each firm $i$ has a capacity of $S^{i}$, and a yield of $y^{i}$. The production process can be thought of as being composed of two steps. In order to have one viable unit of production at the end of the first stage, $1 / y^{i}$ units need to be started at a unit cost of $c_{1}$ per unit started. This corresponds to the idea that only $y^{i}$ percent of the $F P D$ s are usable since panels have to be discarded if any pixels are defective. Following this, further stages of production must be performed on the viable unit at a cost of $c_{2}$ per unit. Thus the total cost of $q^{i}$ units of produced output in the short run for a firm with yield $y^{i}$ and capacity $S^{i}$ is 


$$
\begin{aligned}
T C_{s r}^{i}\left(q^{i}, y^{i}, S^{i}\right) & =\left(\frac{c_{1}}{y^{i}}+c_{2}\right) q^{i} \text { for } q^{i} \leq S^{i} \\
& =\infty \text { for } q^{i}>S^{i}
\end{aligned}
$$

Such firms have a marginal cost of $\frac{c_{1}}{y^{i}}+c_{2}$. Profits are thus

$$
\begin{aligned}
\Pi_{s r}^{i}\left(q^{i}, y^{i}, S^{i}\right) & =P\left(\sum_{j=1}^{k} q^{j}\right) q^{i}-\left(\frac{c_{1}}{y^{i}}+c_{2}\right) q^{i} \text { for } q^{i} \leq S^{i} \\
& =-\infty \text { for } q^{i}>S^{i}
\end{aligned}
$$

The supply curve for the industry is as depicted in Figure 1. As firms behave competitively, each firm supplies its entire capacity if price exceeds its marginal cost. This permits us to replace $q^{i}$ with $S^{i}$ whenever a firm makes positive profits. It also results in the step function form of supply depicted. Firms take the intersection of demand and supply as the given price, and they maximize their profits accordingly in the short run. As drawn, firms with lower indices have higher yields and correspondingly lower marginal costs. Only firms with indices less than $k+1$ supply as drawn in Figure 1 .

\subsection{The Medium Run}

In the short run, firms take their capacities as given, as well as their yields. In the medium run, they take only their yields as given, and they choose capacities. The cost of an additional unit of capacity is $F^{i}$, but a firm which has a yield of $y^{i}$ will incur a cost of $\left(F^{i} / y^{i}\right) q^{i}$ to get an output of $q^{i}$. Of course, they realize that their choice of capacity affects the price in the market and incorporate such considerations in their decision making. Each firm, therefore, chooses its capacity, $S^{i}$, to maximize its medium run profits, which along with its first order condition are given below. 


$$
\begin{aligned}
\Pi_{m r}^{i}\left(y^{i}, S^{i}, S^{-i}\right) & =P\left(\sum_{j=1}^{k} S^{j}\right) S^{i}-\left(\frac{c_{1}}{y^{i}}+c_{2}\right) S^{i}-\frac{F^{i}}{y^{i}} S^{i} \\
\frac{\partial \Pi_{m r}^{i}\left(y^{i}, S^{i}, S^{-i}\right)}{\partial S^{i}} & =P^{\prime}\left(\sum_{j=1}^{k} S^{j}\right) S^{i}+P\left(\sum_{j=1}^{k} S^{j}\right)-\left(\frac{c_{1}}{y^{i}}+c_{2}\right) \\
-\frac{F^{i}}{y^{i}} & =0
\end{aligned}
$$

Figure 2 depicts the capacity choice which solves (4). Note that if a firm chooses to invest in capacity, its profits in the short run must be strictly positive. ${ }^{12}$ Hence, firms will choose to produce all their capacity in the short run.

As all firms choose their capacities in this manner, the solution to this system looks like a Cournot Nash equilibrium. The equilibrium levels of $S^{i}$ are denoted by $S^{i}\left(y^{1}, y^{2}, \ldots, y^{N}\right)$ for $i=1, \ldots, N$. Notice that firms with higher yields will choose higher capacity levels as one would expect.

\subsection{The Long Run}

In the long run, firms choose their yield levels realizing how this affects the outcome in the short and medium runs. The cost to a firm with yield $y_{0}^{i}$ of raising its yield to level $y^{i}$ is denoted by $R^{i}\left(y_{0}^{i}, y^{i}\right)$. We will assume that this takes a particular functional form given by

$$
R^{i}\left(y_{0}^{i}, y^{i}\right)=z\left(y^{i}-y_{0}^{i}\right)+\theta\left(y^{i}-y_{0}^{i}\right)^{2} .
$$

Note this functional form has the property that even small improvements in yield are costly and that larger improvements become progressively more costly.

12If $P\left(\sum_{j=1}^{k} S^{j}\right)-\frac{c_{1}}{y^{j}}-c_{2}=0$, then the lowest yield firm may produce less than its full capacity. It is easily shown that the medium run equilibrium rules out capacity choices that will not be used in the short run. 
Thus, firm $\mathrm{i}$ in the long run wishes to maximize its long run profits given by:

$$
\begin{aligned}
\Pi_{l r}^{i}\left(y^{i}, S^{i}(\cdot), S^{-i}(\cdot)\right)= & P\left(\sum_{j=1}^{k} S^{j}(\cdot)\right) S^{i}(\cdot)-\left(\frac{c_{1}}{y^{i}}+c_{2}\right) S^{i}(\cdot) \\
& -\frac{F}{y^{i}} S^{i}(\cdot)-z\left(y^{i}-y_{0}^{i}\right)-\theta\left(y^{i}-y_{0}^{i}\right)^{2}
\end{aligned}
$$

The first order condition, after using the envelope theorem, gives:

$$
\frac{c_{1}}{\left(y^{i}\right)^{2}} S^{i}(\cdot)+\frac{F^{i}}{\left(y^{i}\right)^{2}} S^{i}(\cdot)-R^{\prime}(\cdot)+P^{\prime}(\cdot) S^{i}(\cdot) \sum_{j \neq i} \frac{d S^{j}\left(y^{1}, \ldots, y^{n}\right)}{d y^{i}}=0
$$

In addition to the long run first order condition given above, we need a steady state condition for each firm. In the steady state, the yield chosen by each firm must equal its initial yield. The steady state condition for each firm, therefore, consists of Equation (7) where $R^{\prime}(\cdot)$ is evaluated at $y^{i}=y_{0}^{i}$.

\section{Costs and Yields}

In this section, we examine data for costs of production at various yields to get an idea of the importance of yield differences in the industry. Cost data are from Stanford Resources, Inc. (Mently and Castellano 1994; Stanford Resources, Inc.1994). They refer to a portion of the $A M L C D$ market, namely color $T F T-L C D s$ produced in 1994.

In the short run, recall that each firm produces up to capacity, as long as its short run costs fall short of price, with costs given by $\left(\frac{c_{1}}{y^{i}}+c_{2}\right) S^{i}$ if their yield is $y^{i}$. Mently and Castellano report unit costs for final output levels $\left(q^{i}\right)$ between 60,000 and 960,000 panels per year and yields between .1 and .9. These unit cost data include variable costs such as materials, supplies, and equipment, as well as direct and indirect labor, employed in producing color TFT-LCDs. They also include capital investment cost in the form of 
depreciation. ${ }^{13}$ Based on these data (net of depreciation), the following regression was estimated to give us estimates of $c_{1}$ and $c_{2}$ :

$$
T C^{i}=\tilde{c}_{1} \frac{q^{i}}{y^{i}}+\tilde{c}_{2} q^{i}+\epsilon
$$

Coefficient estimates and standard errors are reported in Table 3. The form seems to fit the data well with an $R^{2}$ of .93 .

Note that a two stage cost structure is embodied in this cost function. A fraction $y$ of the starts are non defective at the end of the first stage. The cost of the first stage application is $c_{1}$. Thus, to get one unit past the first stage, $1 / y$ units are needed as starts with a cost of $c_{1} / y$ per non defective unit at the end of the first stage. At the second stage, the cost is $c_{2}$ per unit that goes in. Our estimates suggest that variable cost levels rise sharply with reductions in yield, especially at low yield levels.

Our specification of medium run costs also depends on yields. That is, $T C_{m r}^{i}=\left(\frac{c_{1}}{y^{i}}+c_{2}\right) S^{i}-$ $\frac{F^{i}}{y^{i}} S^{i}$, where the last term reflects capacity acquisition cost. As noted above, Stanford Resources also reports data for capacity cost in the form of depreciation. Since these data are reported for final output levels between 60,000 and 960,000 panels per year and yields between .1 and .9 , we can examine the importance of yields in medium run cost. We estimate a regression of the form

$$
T F C^{i}=\tilde{F} \frac{S^{i}}{y^{i}}+\epsilon
$$

As noted in Table 3, our estimate of $\tilde{F}$ is 51.82 , and the $R^{2}$ is $.99 .^{14}$ Depreciation on flow

13Mently and Castellano report high and low cost figures for unit costs and depreciation. The regressions reported here are based on their high cost figures. Regression results using low costs are similar to those reported here and are available from the authors. For procedures used to compute the unit cost data, see Mently and Castellano. Their depreciation figures are based on straight line depreciation over five years. 14Such a high $R^{2}$ makes us suspect the capacity cost data, in particular, come from accounting procedures which replicate our model. 
capacity costs and hence plant costs thus also depend on yield.

Taking both estimates into account, it appears that plants with yields of $30 \%$ or more are viable at 1994 prices. That is, with a yield of $30 \%$, variable costs are about $\$ 710$ per unit and capacity costs are approximately $\$ 170$, while the December 1994 price was around $\$ 1000$. With a $20 \%$ yield, variable costs are about $\$ 790$ per unit, and capacity costs are about $\$ 260$ per unit. In our model, a firm with this yield would show negative medium run profits at the 1994 price.

\section{Simulation Results}

In this Section, we focus on policy experiments in a simple two firm version of our model. We do this for several reasons. First, while the cost equations in our model show a good fit with the data, other data and/or elasticity estimates needed to calibrate the model are not publicly available. Second, the model's equilibrium conditions, especially those for long run equilibrium given by (6) and (7), are highly non linear. This makes it difficult to solve the system analytically with many asymmetric firms. We therefore simulate a simple two firm system to obtain some insights into policy issues.

We focus on two policy experiments. In the first, we look at the effects of a $25 \%$ permanent subsidy to $\mathrm{R} \& \mathrm{D}$ or to capacity acquisition. In the second, we look at the effect of a given one time subsidy on either R\&D or on capacity. In both experiments, we show how the results depend on the degree of asymmetry between the firms.

The model is the same as in the previous sections. For simplicity, we assume a linear form for the market demand curve, which is parameterized as

$$
P\left(\sum_{j=1}^{2} S^{j}\right)=a-b\left(\sum_{j=1}^{2} S^{j}\right)
$$


Short run profits are given by (2), medium run profits by (3) and long run profits by (6). In each of the simulations we look at the evolution towards the steady state of key endogenous variables, including yields, capacity, price, and profits. Our purpose in carrying out these simulations is to help understand the possible consequences of proposed subsidies to $R \& D$ and to volume production in the flat panel industry. Will subsidies to capacity acquisition help or hinder the long run competitiveness of the targeted firm? Are such subsidies likely to raise or reduce welfare?

\section{$5.1 \quad$ A 25\% Subsidy}

We first consider the case of two identical firms. We trace the behavior of the simulated market over time under three scenarios. The first is that of no policy. The second is that of a $25 \%$ subsidy on capacity for one firm, call it firm $A$, so that $F$ is reduced by $25 \%$ for firm $A$ alone. The third is that of a $25 \%$ subsidy on $\mathrm{R} \& \mathrm{D}$ expenditure which corresponds to a $25 \%$ reduction in $z$ and $\theta$ for firm $A$. We then repeat the above three simulations for the asymmetric case where firm $A$ has a lower yield than firm $B$. This allows us to examine the effects of an initial disadvantage on the results of the policy experiments.

In each case, the parameters we use are as follows. The slope of the inverse demand curve, $b$, and its intercept, $a$, are set at $b=1$ and $a=37$. The marginal costs in the short run are given by $\left(\frac{c_{1}}{y^{i}}+c_{2}\right)$ for firm $i=A, B$. We set $c_{1}=c_{2}=1$. In the symmetric case both firms have the same yield of .5 so that $y^{A}=y^{B}=.5$. In the asymmetric case $y^{A}=.4$ and $y^{B}=.5$. The cost of obtaining a unit of capacity is $F$ and is common to both firms. We set $F=2$ throughout. The cost parameters in yield increasing $\mathrm{R} \& \mathrm{D}$ are $z$ and $\theta$, and these are common to both firms and set at $z=120$ and $\theta=1000$. 


\subsubsection{The Symmetric Case}

In the symmetric case, given yields of .5 each, both firms choose a capacity of 10 . The market price to start with is 17 , short run marginal cost of each firm is 3 , and short run profits are 140 each. Capacity costs are 40 per unit and medium run profits are 100 . We run the system through 30 iterations. At each iteration, yields rise, capacity rises, profits rise, and price falls. Yields rise to .583 , which is close to the steady state level of .586 . Capacity rises to 10.28 , while price falls to 16.43 . On the other hand, expenditure on yield-improving $R \& D$ falls dramatically to .06 from a starting value of 2.12 .

When we reduce the cost of capacity acquisition by $25 \%$ to firm $A$ the immediate response in the first iteration is an increase in the chosen capacity to 10.67 . In response to this, $B^{\prime} s$ capacity choice is to keep capacity slightly below 10 . In addition, $A$ invests less in yieldimproving $\mathrm{R} \& \mathrm{D}$ than before and has a lower yield as a consequence! While $B$ invests more than $A$, its investment in $\mathrm{R} \& \mathrm{D}$ also falls from that with no policy in response to $A^{\prime} s$ reduction in R\&D. Initially $A$ invests 1.12 and $B$ invests 1.85 . Both of these numbers are below those which would occur in the absence of policy.

The impact effect of the subsidy is to raise $A^{\prime} s$ gross profits and lower $B^{\prime} s$. Over time, $A$ 's profits rise but then begin to fall while $B^{\prime} s$ rise throughout. The reason for this is that a capacity subsidy (which reduces $F$ ) encourages capacity investment as is evident from (4). However, incentives to do $\mathrm{R} \& \mathrm{D}$ are also diminished by the subsidy on capacity costs. The reason is apparent from the first order condition given in (7). This equation can be thought of as choosing $y_{i}$ on the part of firm $i$ to equate the marginal benefit of yield improving $R \& D,\left(\frac{c_{1}}{\left(y^{i}\right)^{2}} S^{i}(\cdot)+\frac{F^{i}}{\left(y^{i}\right)^{2}} S^{i}(\cdot)+P^{\prime}(\cdot) S^{i}(\cdot) \sum_{j \neq i} \frac{d S^{j}\left(y^{1}, \ldots, y^{n}\right)}{d y^{i}}\right)$, with its marginal cost, $R^{\prime}($.$) . If$ the problem is well behaved we can think of the marginal benefit as decreasing in $y^{i}$. The marginal cost is increasing in $y^{i}$ for our specification, starting from any given initial yield. 
Recall that a capacity subsidy reduces $F$ by $25 \%$. This shifts the marginal benefit curve of doing yield reducing $R \& D$ inwards, reducing the chosen yield.

In contrast, a subsidy to $R \& D$ reduces $R^{\prime}($.$) by 25 \%$ in (7). This shifts the marginal cost of yield reducing R\&D down and out, raising the chosen level of $y^{i}$. This in turn reduces the marginal cost of a unit of capacity as seen in (4) and this raises capacity acquisition in the medium run.

At the end of 30 iterations, $A$ has a yield of .544 , while $B$ has a yield of .579 , both of which are less than that without policy! $A$ 's capacity exceeds that of $B$ (10.68 versus 10.08$)$, and price falls to 16.25 . R\&D expenditure falls to .002 for $A$ and .013 for $B$, both of which are lower than they would be without the policy. Profits in the short run initially go to 142.6 for firm $A$ and 133.8 for firm $B$ as a direct consequence of the higher capacity induced by the subsidy. After 30 iterations, short run profits go to 143.2 and 136.2 , respectively.

In contrast, an $R \& D$ subsidy of $25 \%$ results in an impact effect which almost doubles $A$ 's $R \& D$ expenditure, which goes to 4.07 while $B$ 's goes to 2.06 . Both firms' $R \& D$ expenditures fall steadily over time to .03 and .005 respectively after 30 iterations. Firm $A$ 's capacity rises steadily over time to 10.8 , while $B$ 's stays at about 10 . Yields also rise steadily to .692 and .576 for $A$ and $B$, respectively. $A$ 's short run profits rise throughout while $B$ 's fall throughout ending up at 148.7 and 134.2 , respectively, after 30 iterations. Price falls to 16.15 at the end of 30 iterations.

It is perhaps easiest to compare the effects of the three policies on yields by looking at Figure 3. Each point is an iterative value of yields for A and B. Yields are always increasing. In the first iterations, there are large changes in yields, but these get smaller over time, as depicted by the dots moving closer together as yields rise. The $R$ curve, which gives the yields with the $R \& D$ subsidy, always lies to the right of the $N$ curve, which depicts the yield 
with no policy. The $\mathrm{C}$ curve always lies to the left of the $N$ curve. Thus, while $B$ reaches roughly the same yield across the three scenarios, $A$ 's yields vary considerably, being lowest in the capacity subsidy case and highest in the $R \& D$ subsidy case. Figures 4 and 5 depict the capacity and short run profit paths as a function of time for the two firms across the three scenarios.

\subsubsection{The Asymmetric Case}

While the simulations above provide useful insights, it is important to ask about the extent to which they are modified by firm $A$ being at an initial disadvantage. To cast some light on this, we repeated our simulations for starting values of yields of .4 for $A$ and .5 for $B$. Without any policy, $A$ 's initial disadvantage is slowly overcome. $A$ invests significantly more in $R \& D$ than $B$ and the two firms arrive at the symmetric steady state as before. When a capacity subsidy of $25 \%$ is given, the same kind of effects are obtained as in the symmetric case. $A^{\prime} s$ investment in $R \& D$, while remaining above $B^{\prime} s$, falls due to the subsidy. However, even after 30 iterations, the yield of firm $A$ remains below that of firm $B, .543$ versus .579 ! In contrast, with an $R \& D$ subsidy, $A^{\prime} s$ yields surpass those of $B$ after only 7 iterations. By the end of 30 iterations, the yields are at .691 and .576 , respectively.

Thus, asymmetries in the initial yields only seem to magnify the differences in the two policies. With initial asymmetries, firm $A$ needs to invest heavily in $R \& D$ to catch up with firm B. A capacity subsidy reduces the incentive to do $R \& D$, and as a result, $A^{\prime} s$ yield remains below $B^{\prime} s$. By contrast, an $R \& D$ subsidy enhances the incentives to do $R \& D$; and, $A^{\prime} s$ yield, short run profits, and capacity overtake $B^{\prime} s$ in a small number of periods. The behavior of yields in the asymmetric case are depicted in Figure 6. Again, each point is an iterative value of yields for A and B. Yields are always increasing, and at first, there are large changes in yield, but these get smaller over time, as depicted by the dots moving closer 
together as yields rise. The $R$ curve, which gives yields with the $R \& D$ subsidy, always lies to the right of the $N$ curve, which depicts the yield with no policy. The $\mathrm{C}$ curve always lies to the left of the $N$ curve. Again, while $B$ reaches roughly the same yield across the three scenarios, $A$ 's yields vary considerably, being lowest in the capacity subsidy scenario and highest in the $R \& D$ subsidy scenario. Figures 7 and 8 depict the capacity and short run profit paths as a function of time for the two firms across the three scenarios in the asymmetric case.

\subsection{A Given Subsidy Expenditure}

The results so far suggest that $R \& D$ subsidies seem to be more effective than capacity subsidies. The reason is that a $R \& D$ subsidy raises yields, which in turn effectively reduces the cost of a unit of capacity, and encourages capacity acquisition, while a subsidy on capacity raises the incentive to do capacity acquisition, but reduces the incentive to do yield improving R\&D.

The simulations so far compare a given permanent (25\%) subsidy on $R \& D$ versus one on capacity. They do not compare the effects of a given, one shot expenditure on the two kinds of subsidies, which is, of course, the relevant comparison for the allocation of given funds to alternative policies. Such a simulation would also address another criticism of the DoD initiative: namely that it is too small to produce the desired effect. It is obvious that a dollar spent on $R \& D$ or capacity subsidies need not result in exactly a dollar increase in gross expenditure. It could result in more than a dollar increase in gross expenditure, so that net expenditure (expenditure net of the subsidy) rises, or it could result in less than a dollar increase in gross expenditure, so that net expenditure falls!

First note that in our earlier simulations, yields and capacity stabilize as the steady state is reached, so that $R \& D$ and capacity expenditures fall over time. If we simulate the effects 
of a one time subsidy, the impact or immediate effect will be to raise the gross level of the targeted variable above its no policy path, but when the subsidy is removed in the next period, these higher levels can not be sustained. In the model we use, there is no effect of a one shot policy on the steady state. However, this does not mean that these policies have no effect: the path to steady state is very different under the two policies.

Figure 9 plots the net effect of a $\$ 1$ subsidy (applied as the equivalent ad-valorem level) when applied to $R \& D$ and to capacity in the symmetric case. The dashed line in the figure plots the difference between the targeted firm's $R \& D$ expenditure (net of the government subsidy) when it receives a one-shot dollar subsidy for $R \& D$ and its $R \& D$ expenditure without policy. The cross-hatched line plots the difference in the firm's capacity expenditure (net of the subsidy) with and without this $R \& D$ subsidy. The solid and dotted lines have the same interpretations when the policy is a one-shot subsidy for capacity acquisition. We focus on these plots since the path of yields and capacity can be inferred from them.

A dollar spent on $R \& D$ results in an immediate increase in gross expenditure on $R \& D$ of about 2.62 dollars, so that net expenditure rises by about 1.62 dollars as shown in Figure 9. Of course, in the following period, expenditure on $R \& D$ falls below that with no policy. However, this reduction is only about .4 , so that the impact effect dominates. This results in the path of yields lying above the no policy path. Moreover, the impact effect of the $R \& D$ subsidy on capacity expenditure is to raise it above its no policy path as shown in Figure 9. This is because, as discussed earlier, higher yields reduce the cost of investing in capacity and so result in a greater incentive to invest in capacity. Thus, capacity also lies above its no policy path in this case.

In contrast, a dollar spent on a capacity subsidy results in an impact effect of a net reduction in expenditure on capacity acquisition of about -.76 ! In other words, gross ex- 
penditure rises by only .24 as there is crowding out of private expenditure by the government subsidy. In addition, expenditure on $R \& D$ falls as a capacity subsidy reduces the incentive to invest in $R \& D$ as argued earlier. In subsequent periods there is a small positive effect on $R \& D$ and capacity expenditures relative to the no policy path which fades out over time with the capacity expenditure becoming negative in some later periods.

Figure 10 plots the net effect of a $\$ 1$ subsidy (applied as the equivalent ad-valorem level) when applied to $R \& D$ and to capacity in the asymmetric case with firm $A$ 's yield reduced from .5 to .3 . The four curves have the same interpretation as in Figure $9 .^{15}$ First, note that a dollar spent on $R \& D$ results in an immediate increase in gross expenditure on $R \& D$ of only 1.68 dollars, so that net expenditure rises by about .68 dollars, as shown by the dashed line in the figure. To understand this, recall that with asymmetric firms, if the laggard (firm $A$ ) does not drop out, it spends more on $R \& D$ than the firm which is ahead in order to catch up. With a convex $R \& D$ cost function, the more $R \& D$ that is done, the more expensive is further $R \& D$. This, plus the fact that steady states are independent of initial conditions as long as the firms remain in the market, make a given dollar subsidy on $R \& D$ translate into a smaller equivalent percentage in the asymmetric case (as compared to the symmetric one). While both factors reduce the effectiveness of $R \& D$ subsidies in the asymmetric case, the qualitative conclusions drawn in the symmetric case remain. As before, capacity acquisition is encouraged by the $R \& D$ subsidy, and in subsequent periods $R \& D$ expenditure falls below that with no subsidy at all.

A capacity subsidy of a dollar initially reduces and then raises $R \& D$ expenditure. Net expenditure on capacity falls by about .61 as the gross expenditure rises by only .39 . In contrast to the symmetric case, however, expenditure on capacity does not rise above the no policy level in subsequent periods. These simulations thus suggest that $R \& D$ subsidies 15The intermediate case where firm A's yield is .4 shows effects similar to these. 
dominate capacity subsidies if the aim is to raise yields and capacity. They also suggest that R\&D subsidies have greater leverage in the symmetric case as they have a larger net impact effect under these circumstances.

\section{Concluding Remarks}

The public debate on flat panels has focused largely on whether the Clinton administration initiative is clever promotion of dual use technology or straightforward industrial policy. In contrast, we examine whether subsidies to promote volume production ( $\$ 199$ million of the initiative) or R\&D subsidies ( $\$ 318$ million of the initiative) are more effective. Our results suggest that $R \& D$ subsidies provide the government more leverage in supporting an industry. In the context of our model, subsidies for capacity can, in fact, backfire because they reduce a firm's incentive to invest in yield-improving $R \& D$. As always, these results should be interpreted in the context of the model and its exclusions. For example, the inclusion of learning by doing would make our distinction between $R \& D$ and capacity subsidies less clear. Perhaps the most interesting revision would be to see how the inclusion of forward looking behavior by firms would effect our results with and without policy.

Our model can be used to examine other issues, such as the effects of entry. The effect of entry is important for the flat panel industry, not only with respect to entry of U.S. firms, but also with respect to recently announced capacity increases of Korean firms (Pollack 1995). It is entirely possible that profits are not eroded to zero by entry because entrants use technologies with lower yields than incumbent firms who have done the necessary $R \& D$ to maintain higher yields. ${ }^{16}$

An additional issue of interest is how the initial configuration of firms' capacities and

16Hence, new firms have disincentives for entry. Incumbent firms realize that raising capacity will reduce price and this results in their voluntarily limiting their capacity. Thus, it is likely that despite firms choosing output competitively, profits are non zero due to quasi rents that arise. 
yields determines final industry configuration. Indeed, we suspect that firms which enter early can remain and earn profits later on when new entry is not viable! These issues, as well the effect of $R \& D$ or capacity subsidies on the final configuration of firms and the minimum size of subsidies necessary to accomplish particular goals, are left for future work. 


\section{References}

Baldwin, Richard and Paul Krugman. 1988. Market access and international competition: a simulation study of $16 \mathrm{~K}$ random access memory. In Robert Feenstra, ed. Empirical Methods for International Trade. Cambridge: MIT Press.

1989. Persistent trade effects of large exchange rate shocks. The Quarterly Journal of Economics 104: 635-654.

Barfield, Claude E. 1995. Flat-panel initiative: A bad idea. Issues in Science and Technology (Spring):9-12.

Barfield, Claude E. 1994. Flat panel displays: A second look. Issues in Science and Technology (Winter):21-25.

Davis, Bob. 1995. Clinton's key technology-policy effort is dealt a blow by Congressional study. The Wall Street Journal (Wednesday, May 3):A2, A6.

Edmondson, Gail and Neil Gross. 1995. The grand alliance in flat panels. Business Week (August 28):73.

Flamm, Kenneth S. 1995. In defense of the flat-panel display initiative. Issues In Science and Technology (Spring):22-25.

Flamm, Kenneth S. 1994. Flat-panel displays: Catalyzing a U.S. industry. Issues in Science and Technology (Fall):28-32.

Hamilton, David P. 1995. An idea: Replace light bulbs with LEDs. The Wall Street Journal (May 10):B1.

Jensen, Richard and Marie Thursby. 1987. A decision theoretic model of innovation, technology transfer, and trade. The Review of Economic Studies (LIV): 631-647. 
MacKnight, Susan. 1994. Flat panel displays: Redefining the parameters of U.S. government support for industry? Japan Economic Institute Report No. 29A. Washington, D.C.: Japan Economic Institute.

Mentley, David E. 1995. Financial analysis of the TFT industry. In International Display Report (June 13). Mountain View, CA: SEMI Newsletter Service.

Mentley, David E. 1994a. Forecast inflation. In International Display Report [November 15]. Mountain View, CA: SEMI Newsletter Service.

Mentley, David E. 1994b. Flat panel displays for military airplanes. In International Display Report (July 15). Mountain View, CA: SEMI Newsletter Service.

Mentley, David E., and J. A. Castellano. 1994. Flat panel display manufacturing cost: A comparative analysis. 2nd edition. San Jose, CA: Stanford Resources, Inc.

Miller, Debra L. 1995. Forum: Flat-panel displays. Issues in Science and Technology: 8 Mowery, David C. 1995. Forum: Flat-panel displays. Issues in Science and Technology: 8.

Nakamoto, Michiyo, and Alice Rawsthorn. 1995. Japanese race to develop wall-hung plasma TVs. Financial Times (Friday, August 25):3.

Patton, Robert, and Alice Rawsthorn. 1995. A wall-hanging wonder. Financial Times (Friday, September 1):8.

Pollack, Andrew. 1995. Japanese face glut of computer screens. The New York Times (May 29):A39.

Rosenbloom, Richard S., and Michael A. Cusumano. 1987. Technological pioneering and competitive advantage: The birth of the VCR industry. California Management Review 29 (4):51-76.

Schott, Jeffery J. 1994. The Uruguay round: An assessment. Washington, D.C.: Center 
for International Economics.

Stanford Resources, Inc. 1994. World average selling prices TFT-LCDs used in portable computers [chart]. In The proceedings of the 11th annual flat information display conference and exhibition. San Jose, CA: Stanford Resources, Inc.

Tyson, Laura D'Andrea. 1992. Managing trade and investment: Europe's evolving strategy in the electronics industry. In Trade conflict in high-technology industries. Washington, D.C.: Center for International Economics.

U.S. Department of Defense. 1994. Building U.S. capabilities in flat panel displays. Final report. DOD. 


\begin{tabular}{|c|c|c|}
\hline \multicolumn{3}{|c|}{$\begin{array}{c}\text { Table } 1 \\
\text { Commercial FPD Sales }\end{array}$} \\
\hline & 1993 & $2000^{*}$ \\
\hline Total Value (in Billions) & $\$ 6.5$ & $\$ 20$ \\
\hline By Technology & $\%$ & $\%$ \\
\hline Non-Emissive (LCD) & 87 & 89 \\
\hline AMLCD & 29 & 55 \\
\hline Other & 58 & 34 \\
\hline Emissive & & \\
\hline Plasma & 4 & 4 \\
\hline Electroluminescent & 1 & 2 \\
\hline Other & 8 & 5 \\
\hline By Application & & \\
\hline Computer & 61 & 67 \\
\hline Consumer & 12 & 14 \\
\hline Business & 9 & 5 \\
\hline Industrial & 12 & 8 \\
\hline Transportation & 6 & 6 \\
\hline
\end{tabular}

*Projections

Sources: DoD 1994, Figures 2-1, 2-7, 3-1, 3-2; Stanford Resources 


\begin{tabular}{||ccccc||}
\hline \hline \multicolumn{5}{|c||}{ Table 2 } \\
& 1993 & Market Shares by Country \\
\hline \hline & LCD & AMLCD & Plasma & Electroluminescent \\
Japan & 92 & 98 & 68 & 47 \\
U.S. & 1 & 1 & 19 & 50 \\
Other & 7 & 1 & 13 & 3 \\
\hline \hline
\end{tabular}

Sources: DoD 1994, Figures 4-1, 4-2 and Table 4-1; Stanford Resources 


\begin{tabular}{|c|c|c|}
\hline \multicolumn{3}{|c|}{$\begin{array}{c}\text { Table } 3 \\
\text { Cost Regressions }\end{array}$} \\
\hline $\begin{array}{l}\text { Dependent } \\
\text { Independent }\end{array}$ & $\begin{array}{c}\text { Total } \\
\text { Variable Cost }\end{array}$ & $\begin{array}{c}\text { Total } \\
\text { Fixed Cost }\end{array}$ \\
\hline$q / y$ & $\begin{array}{l}47.12 \\
(7.03)\end{array}$ & $\begin{array}{l}51.82 \\
(.21)\end{array}$ \\
\hline$q$ & $\begin{array}{l}554.13 \\
(29.09)\end{array}$ & \\
\hline $\mathrm{R}^{2}$ & .93 & .99 \\
\hline Observations & 45 & 45 \\
\hline
\end{tabular}

Standard Errors in Parenthesis

Data: Mently, D. E. and J. A. Castellano 1994. 


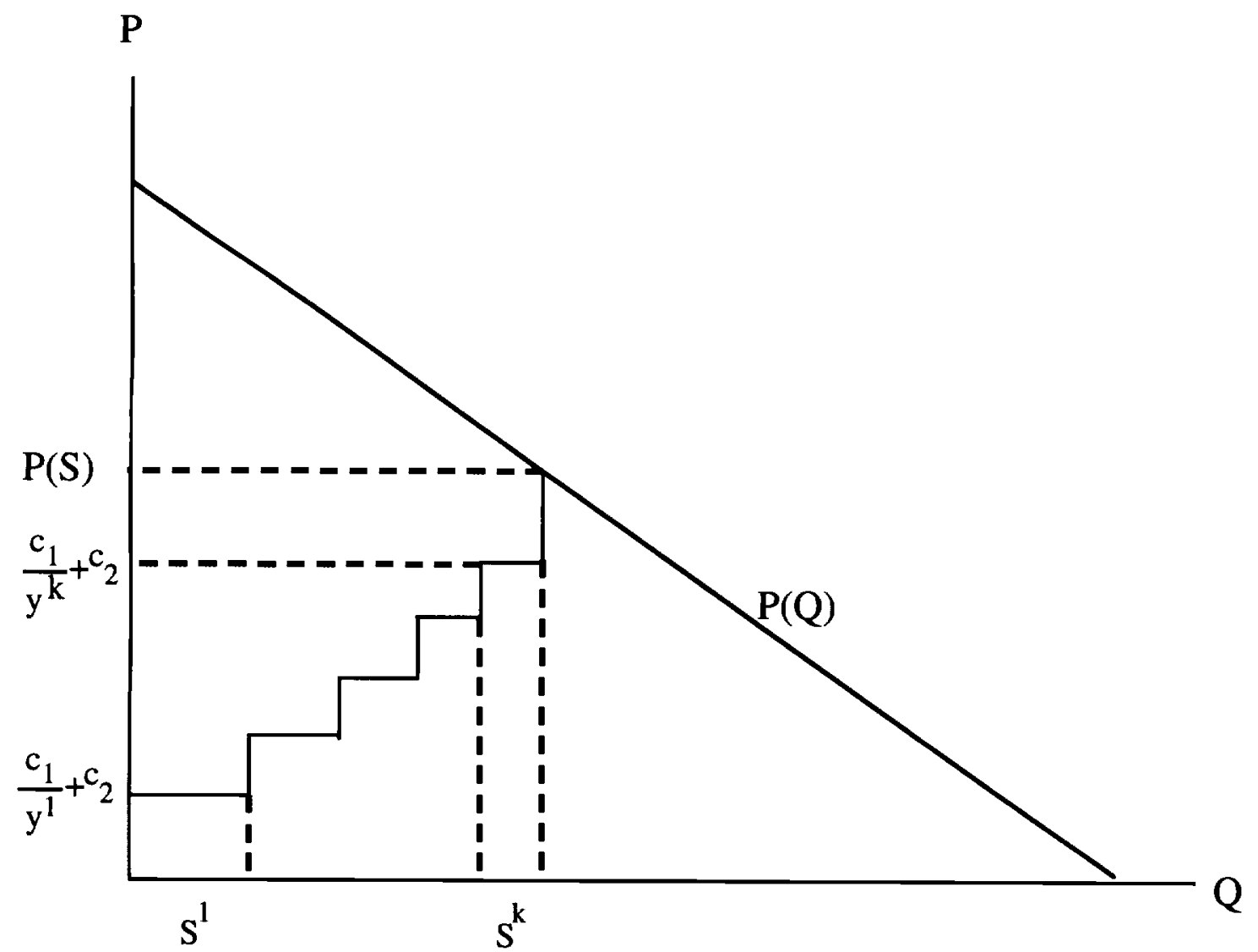

Figure 1. Short run equilibrium. 


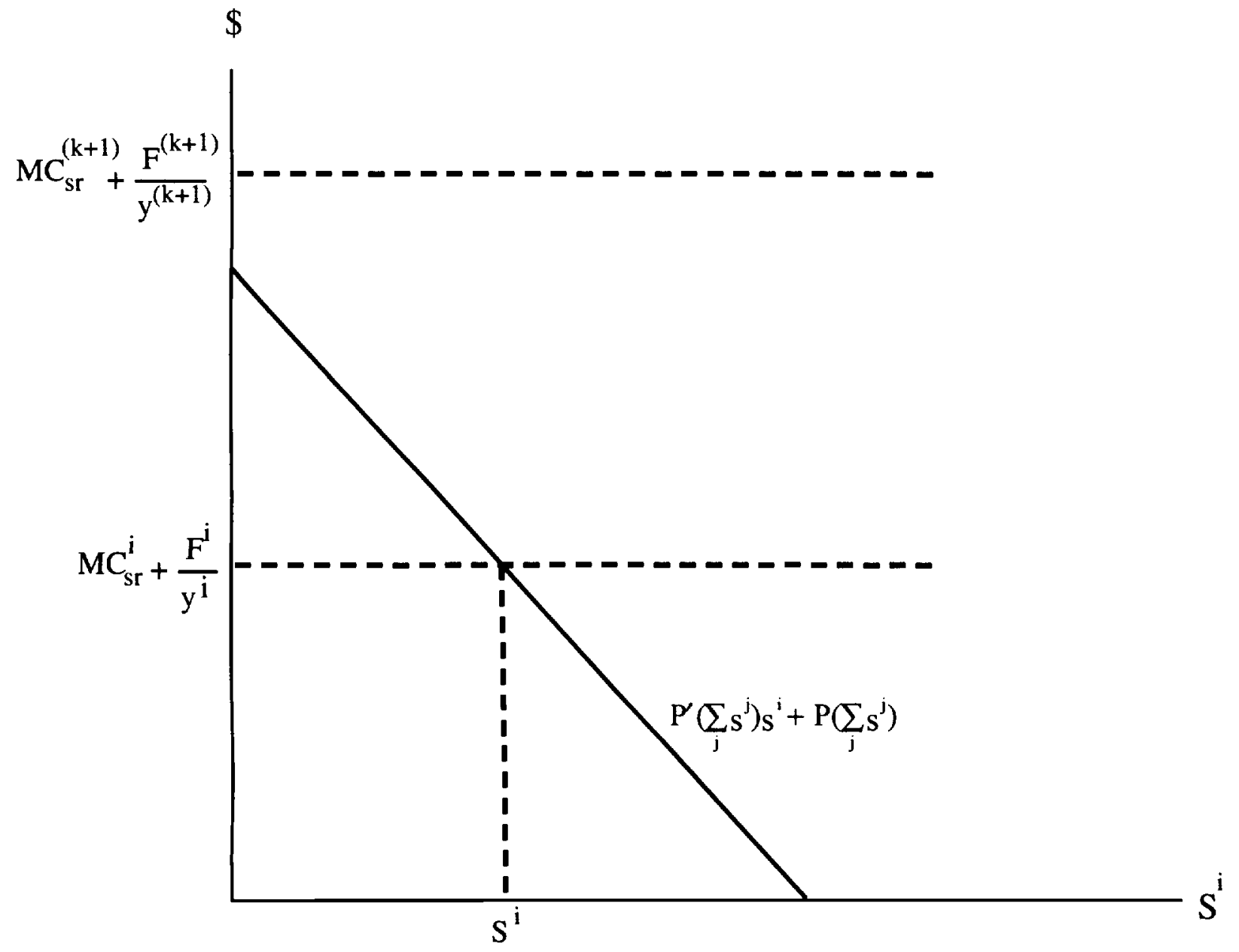

Figure 2. Medium run equilibrium. 


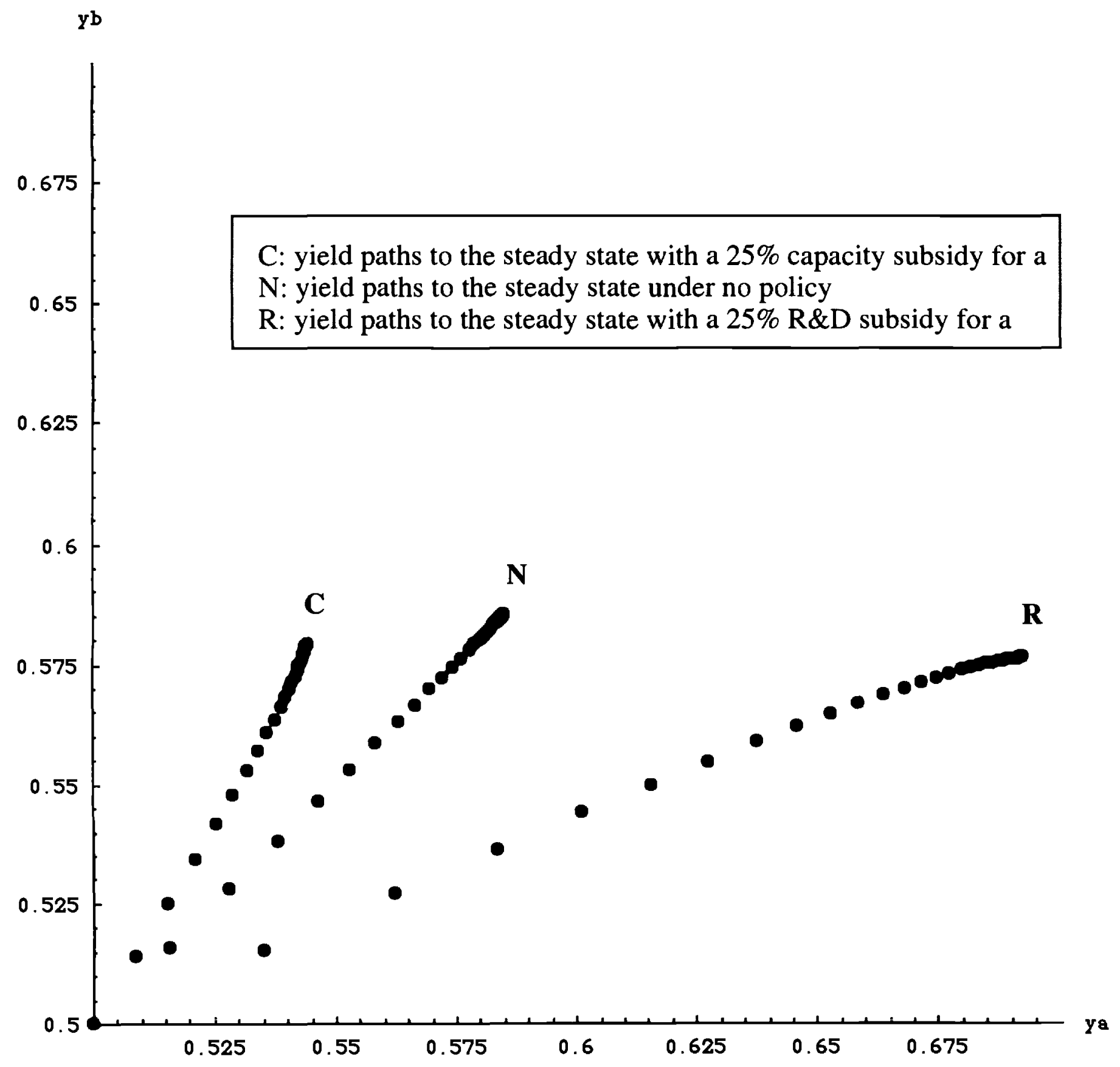

Figure 3. Yield paths to the steady state in the symmetric case with ya $0=.5$ and yb0 $=.5$. 


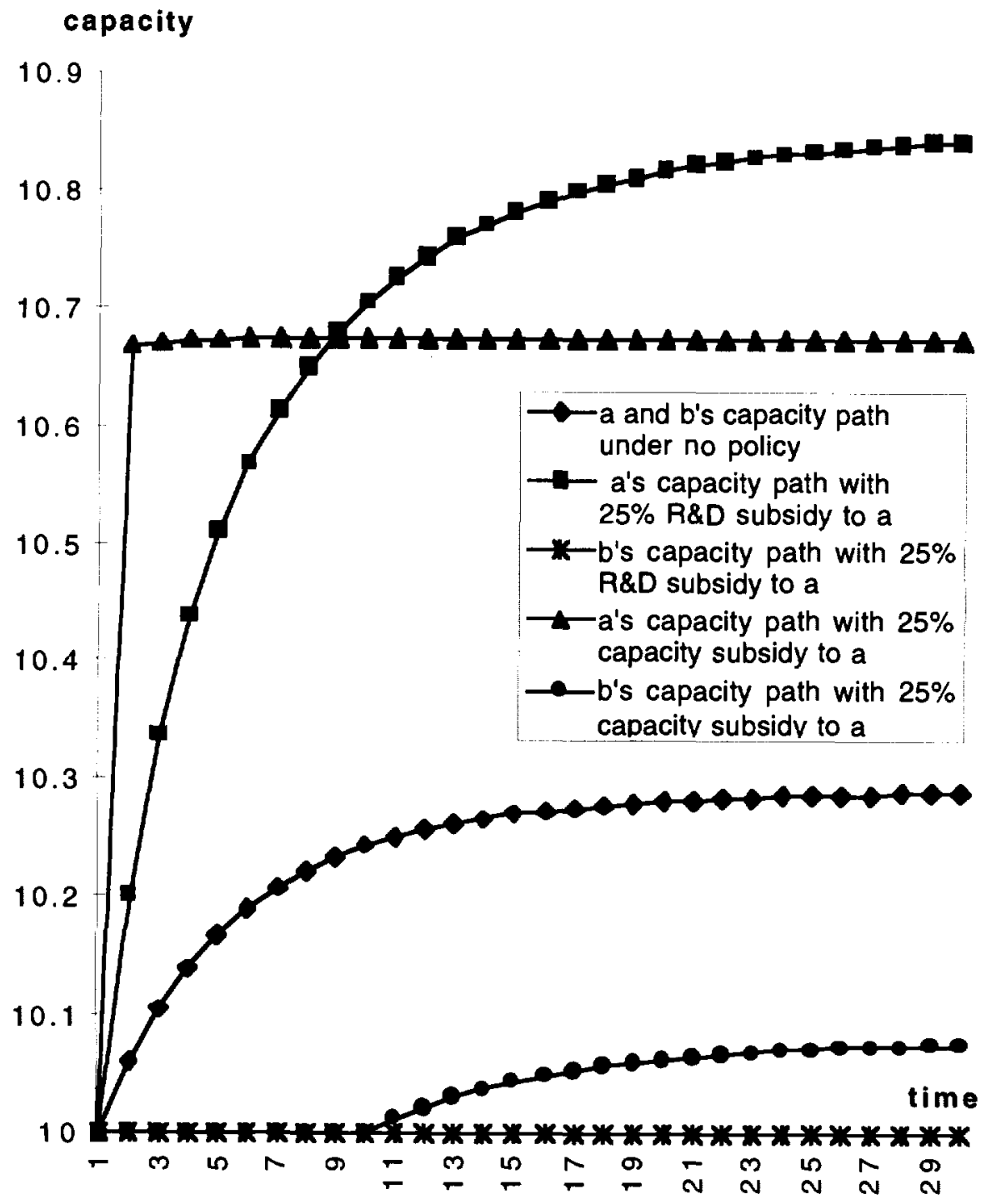

Figure 4. Capacity paths to the steady state in the symmetric case with ya $0=.5$ and $y b 0=.5$. 


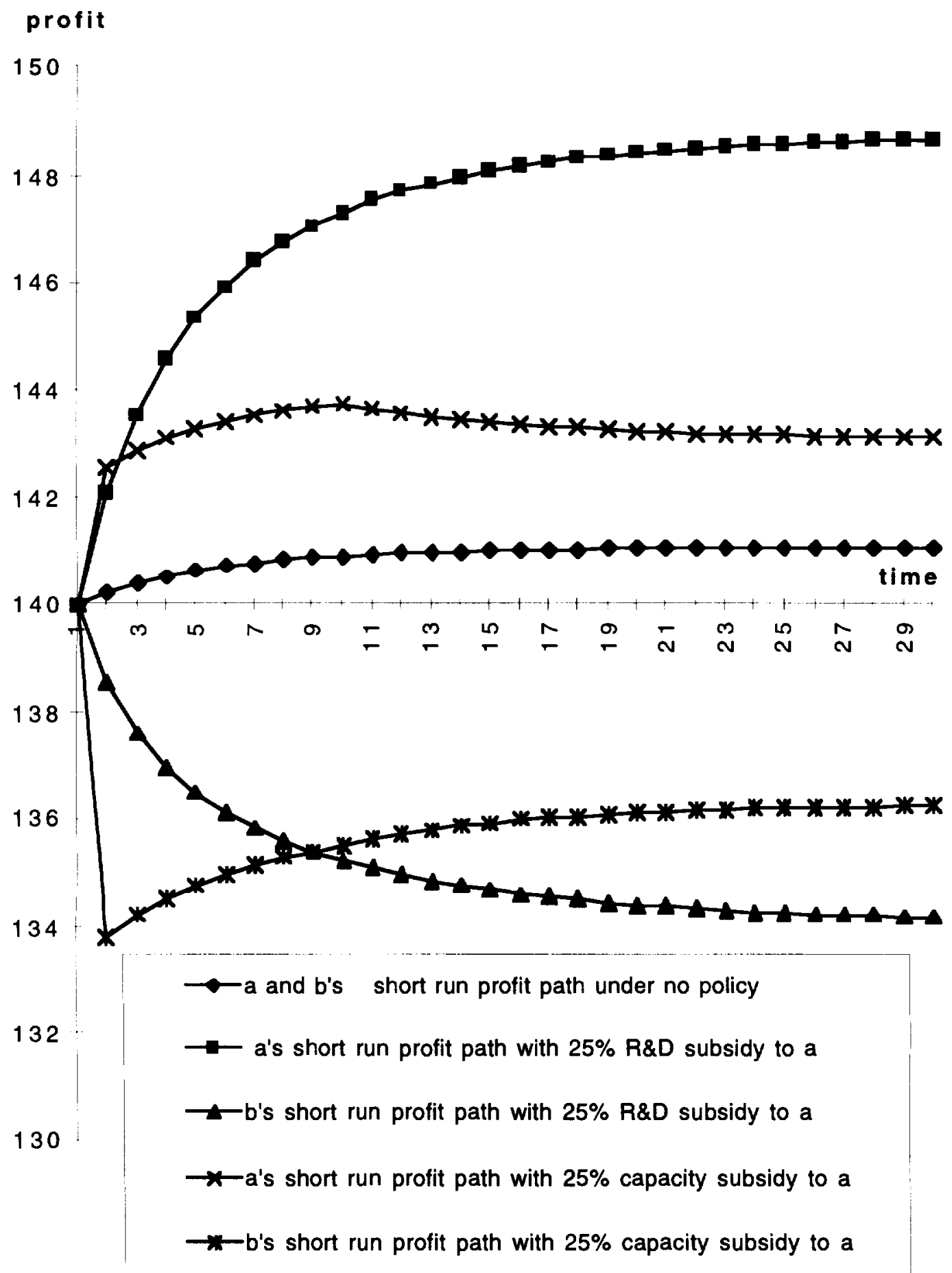

Figure 5. Short run profit paths to the steady state in the symmetric case with ya0 $=.5$ and $y b 0=.5$. 


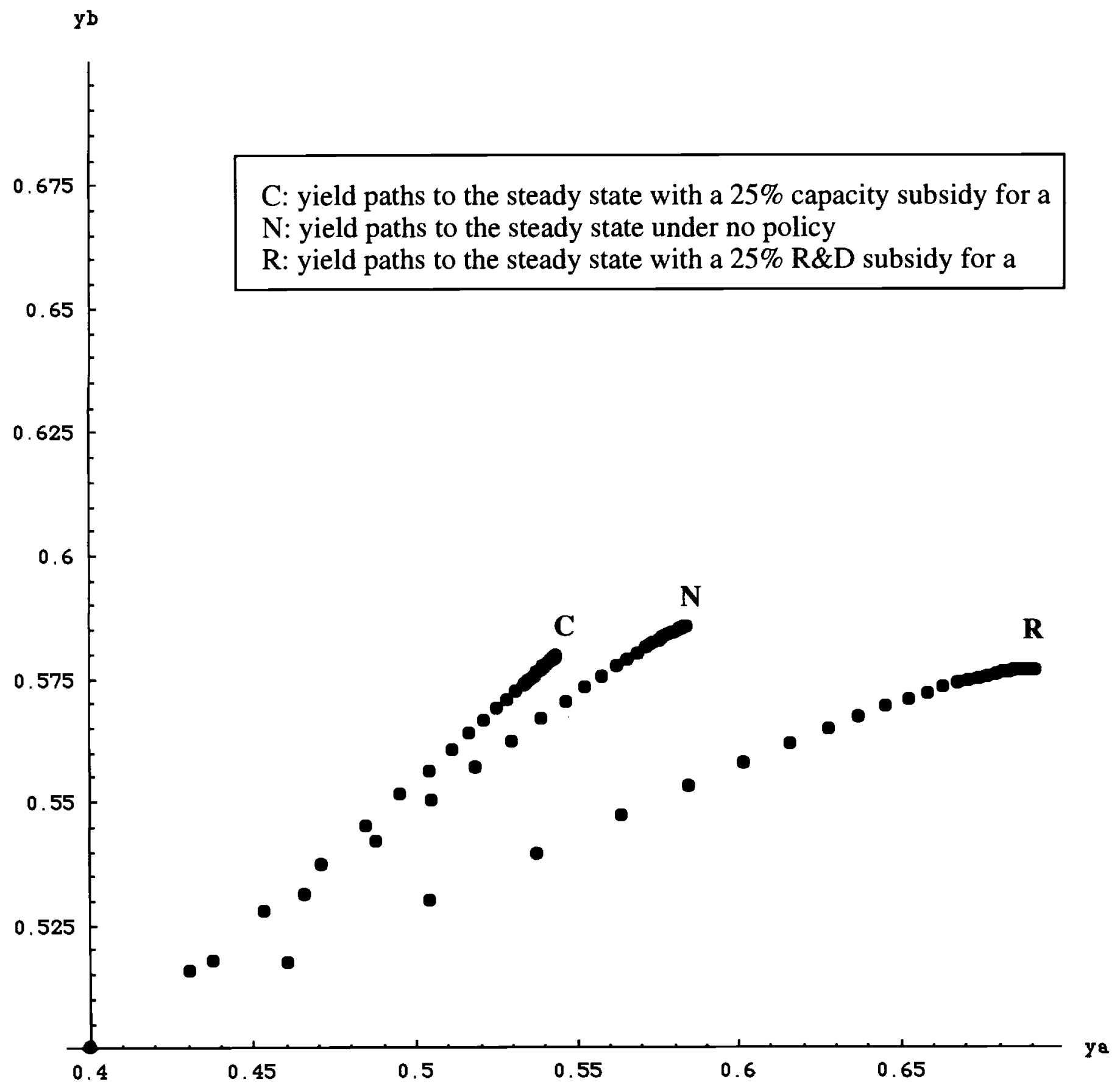

Figure 6. Yield paths to the steady state in the asymmetric case with $\mathrm{ya} 0=.4$ and $y b 0=.5$. 


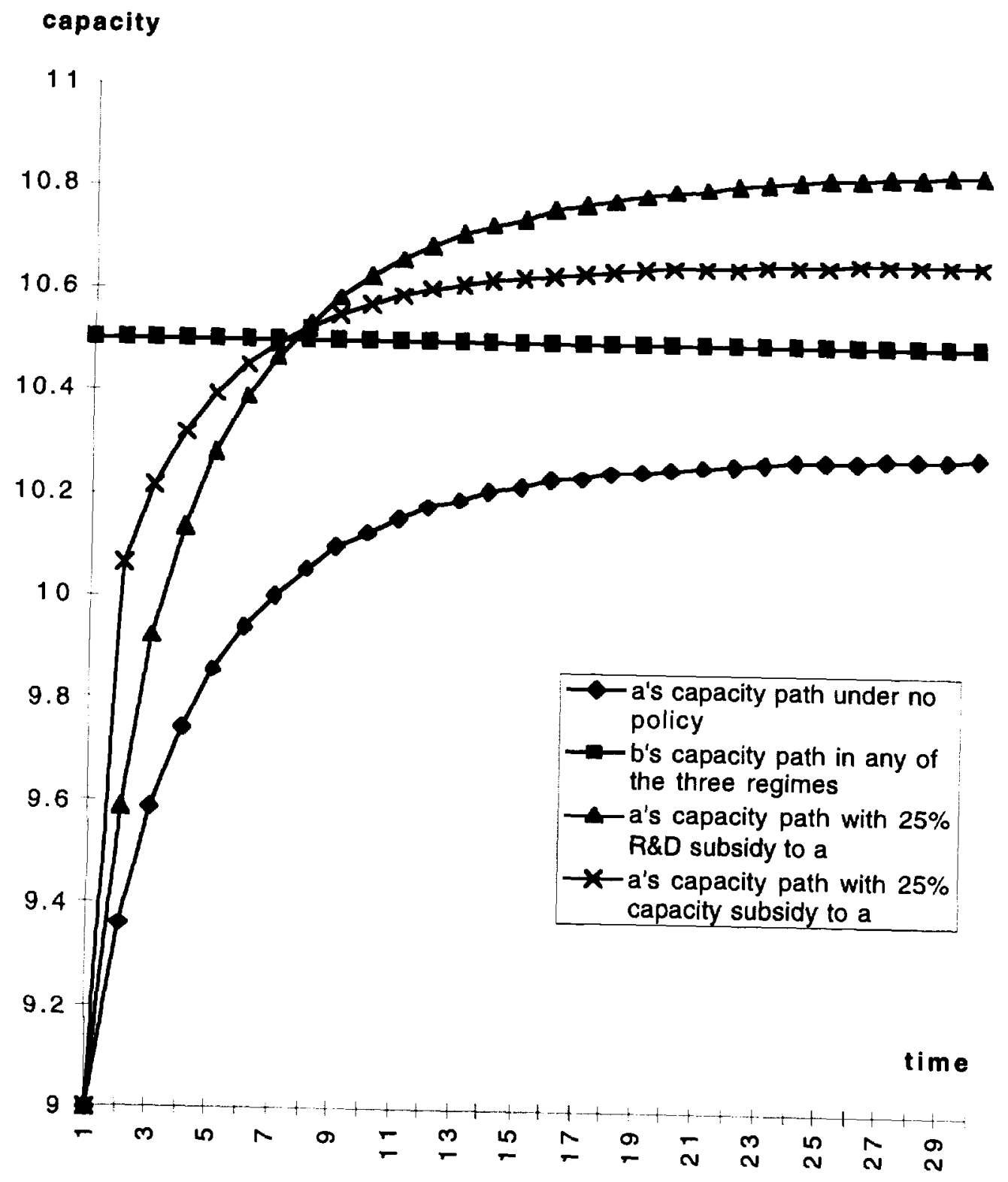

Figure 7. Capacity paths to the steady state in the asymmetric case with $\mathrm{ya} 0=.4$ and $\mathrm{yb0}=.5$. 


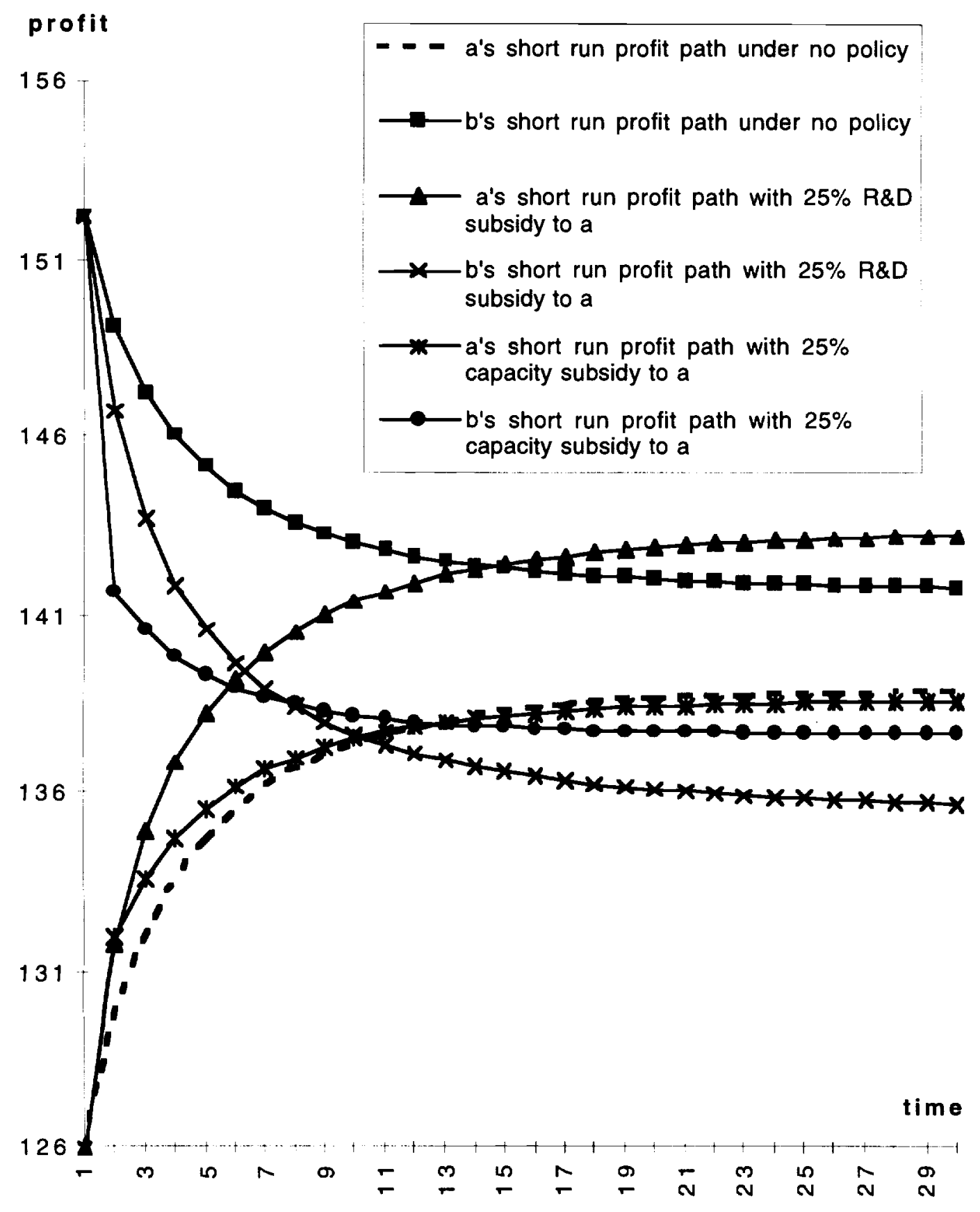

Figure 8. Short run profit paths to the steady state in the asymmetric case with $y a 0=.4$ and $y b 0=.5$. 


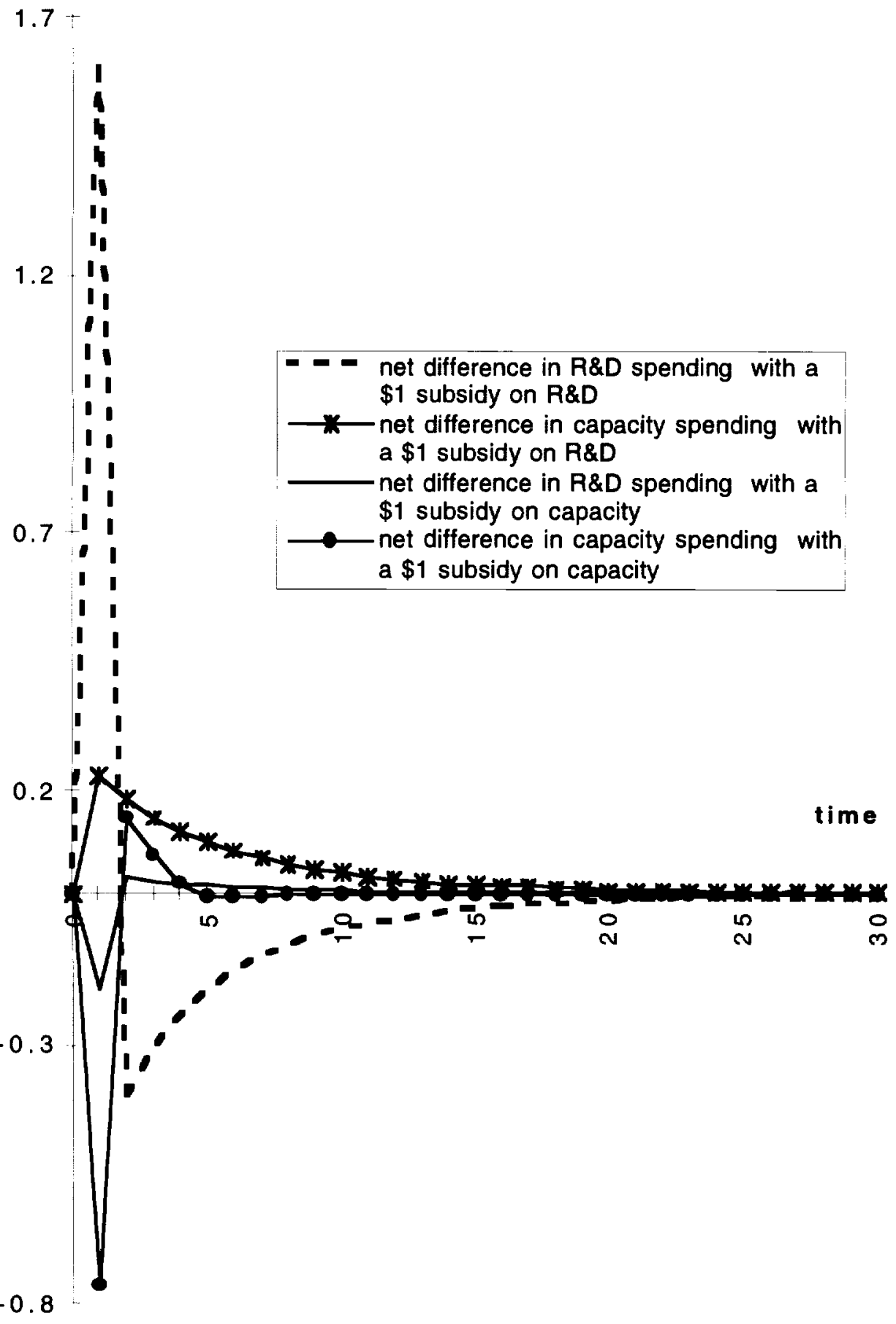

Figure 9. Net effects of $\$ 1$ subsidies for the symmetric case with $y a 0=.5$ and $y b 0=.5$. 


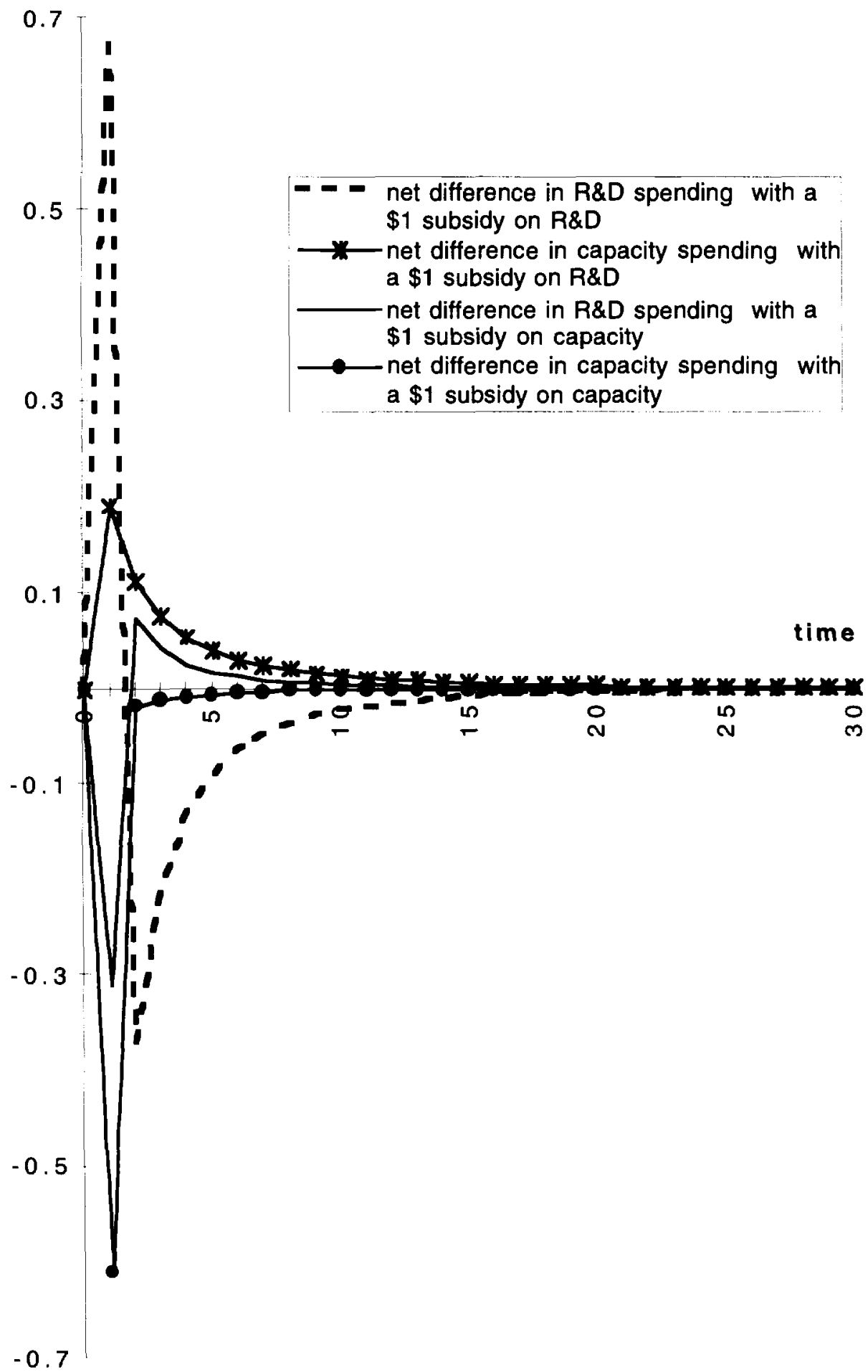

Figure 10. Net effects of $\$ 1$ subsidies for the asymmetric case with $y a 0=.3$ and $y b 0=.5$. 